\title{
Strategies of Theoretical Physics Instruction Reform
}

\author{
Tingting Liu \& Haibin Sun \\ Institute of Physics and Electronic Engineering \\ Taishan University, Tai'an, Shandong 271021, China \\ E-mail:1ttphd@163.com
}

\begin{abstract}
Theoretical physics is the main constitute part of physics science. The instruction of theoretical physics courses plays an important role in the research of basic science and training physics talents. Most of students considered that the knowledge of theoretical physics is very abstract and causing many difficulties in study. But students have initiatives to learn theoretical physics well. Teachers can implement some teaching reform strategies to improve the quality of theoretical physics instruction. The strategies are as follow: stimulate students' interest in learning, perfect students' cognitive structure and knowledge structure of theoretical physics; optimize the system of theoretical physics curriculum, enrich the instruction contents; apply heuristic instruction in theoretical physics teaching; establish virtual theoretical physics experiments and improve assessment and appraisal methods, promote students' all-round development.
\end{abstract}

Keywords: Theoretical physics, Instruction reform, Strategy

This research is supported by the Instructional Reform Key Project of Taishan University (2000905).

\section{Introduction}

Theoretical physics is the main constitute part of physics science. Theoretical physics mainly includes theoretical mechanics, electrodynamics, quantum mechanics, thermodynamics, statistical physics and special theory of relativity, and so on. He indicated that the research of theoretical physics usually brought new theoretical ideas, new guiding principles and new ways for exploring (1993). M. Born has pointed out that the theoretical physics teaching has a threefold aim, since it is concerned with three different groups of students. The first group seeks a general education from theoretical physics. The second group consists of those students who need theoretical methods for professional purposes. The third group consists of the few outstanding students who wish to devote their lives to science itself, in research and higher instruction (1941).

In higher education, theoretical physics courses are the main courses of physics major. The instruction of theoretical physics plays an important role in the research of basic science and training physics talents.

\section{The current teaching situation of theoretical physics curriculum}

We designed the questionnaire of theoretical physics instruction, and questionnaire investigation was carried out in 90 collected physics undergraduate students in grade 2005 and 2006 . The investigation results are as follow.

$76 \%$ of students considered that the knowledge of theoretical physics is very abstract, and causing many difficulties in study. $10 \%$ of students had strong interest in learning theoretical physics, $74 \%$ of them had little interest in learning but want to learn the courses well, and $16 \%$ of them had no interest in learning the courses. The survey indicated that most of the students have a lower learning interest level, and they have initiatives to learn theoretical physics.

$74 \%$ of students considered they learned more knowledge from lecture methods and teaching tutorials. Traditionally, theoretical physics in higher education has been taught by lecture methods. Lecture methods can enable students to feel better prepared for learning and examinations. Some researches have pointed out that lectures are not the most efficient way of promoting learning, but encourage passivity among students and lead to a dependency culture where students become unable to initiate any learning activities without being directed by a lecturer (Mary Stewart, 2002).

On the basis of our teaching practices and students' questionnaire, we considered the reasons for the unsatisfactory teaching effects of theoretical physics courses are: (1) students don't realize the importance of theoretical physics courses; (2) the theory of theoretical physics is more complicated and abstract than general physics; (3) the structures and contents of some theoretical physics textbooks are comprehensive; (4) the class hour of theoretical physics courses are shorter than before; (5) theoretical physics teachers appraise students' 
academic achievements mainly through examination.

\section{Strategies of Theoretical Physics Instruction Reform}

\subsection{Stimulate students' interest in learning, perfect students' cognitive structure and knowledge structure of theoretical physics}

The process of learning physics is that the student individual interacts with the physical environment, the student individual recognizes the physical world, and changes themselves cognitive structure (Wang, 2009). Compared with general physics courses, theoretical physics focus on the abstract theory, use more complicated mathematical knowledge, and demand further logical thinking capability. Theoretical physics courses have higher demand for students' scientific knowledge and thinking ability. Wan considered that the learning effects of theoretical physics courses depend on not only the physical concepts and laws of their own, but also the learning interest and motivation of students (2005). In the long-term teaching practices, the vast majority of students has preconceptions that "theoretical physics is very difficult", and is afraid of the theoretical physics learning. Therefore, the priority of theoretical physics instruction is to stimulate the learning interest of students.

First of all, teachers should grasp the knowledge base and the capability base of students before class teaching, and analyse the confusions and difficulties that may arise in the process of learning.

Secondly, teachers should design the theoretical physics instruction on the basis of characteristics of students and the courses. In the process of theoretical physics instruction, teachers can stimulate students' learning interest by the creation of "cognitive conflict", and solve the cognitive conflict using suitable instruction methods. In the process, teachers can help students understand concepts and laws of physics, develop thinking ability and perfect cognitive structure of theoretical physics.

Thirdly, teachers should enforce the integration of different theoretical physics course, and advance the knowledge structure of theoretical physics. Knowledge is often restructured rather than accumulated. Achievement occurs not only by accruing or accumulating information, but also by the creation of higher-order structures that aid in reinterpreting old information or aid in accounting for new information (Hattie, John, et al, 1998). The integration can reduce the burden of students learning. For example, separation of variables that lectured in the mathematical physics course usually is used in the course of quantum mechanics and electrodynamics. When students grasped the procedure of separation of variables, they can flexible apply the method to solving some similar theoretical physics problems easily.

\subsection{Optimize the system of theoretical physics curriculum, enrich the instruction contents}

The integration and optimization of theoretical physics curriculum can be progressed through the reduction of quantity and the choiceness of quality of the course contents (He Ping, 2000).

Firstly, the reduction of theoretical physics course contents embodied in the class hour, curriculum system and instruction contents. On the basis of selected excellent textbooks, we can modernize the instruction contents of theoretical physics, and add frontier knowledge of theoretical physics to the class instruction on the grounds of the subject characteristic.

Secondly, the choiceness of quality of the course contents should follow some necessary principles, such as: strengthen the systematic of discipline theory; describe the basic concepts and basic laws accuracy; train students with reasonable exercise and homework (Lin, 2000).

Specifically, the measures that we take to optimize theoretical physics instruction contents as follow:

For "Theoretical Mechanics" course, we can cut some contents of Newtonian mechanics that similar to the general physics course, and enhance analytical mechanics instruction. By doing so, we can help students learn to use mathematical analysis methods to study mechanical problems, lay a solid foundation for the follow-up of theoretical physics courses.

For "Quantum Mechanics" course, we concentrate in our course on those features of quantum mechanics that are radically new compared with classical mechanics (Muller R. and Wiesner H., 2002). Therefore, we cut some contents that similar to the atomic physics course; add some contents such as spin, second quantization, Hilbert space and representation. We can emphasize the development of quantum mechanics theory in the instruction process, and help students to avoid classical misconceptions and help students to construct a proper quantum mechanical understanding.

For "electrodynamics" course, we arrange the instruction contents around the Maxwell Equations and Lorentz force, increase some general knowledge of quantum electrodynamics, introduce the applications of basic principles of electrodynamics in science and technology, strengthen the education of scientific methods, and 
integrate the instruction of electrodynamics and quantum mechanics.

For "Thermodynamics and Statistical Physics" course, we reduce some contents that similar to the thermology course, and strengthen the instruction of statistical physics.

\subsection{Apply heuristic instruction in theoretical physics teaching}

In theoretical physics instruction, teachers should use integrated various teaching methods in order to achieve the best teaching effects.

We apply heuristic teaching method and discussional teaching method, guide students to carry out inquiry learning (Sun \& Gong, 2007). While teaching, teachers should pay attention to teaching interaction. Teachers can conduct students to take the original problem as a starting point and rethink their predecessors understanding and problem-solving methods. Teachers should encourage students to dare to query the conclusions of any existing, and make further explore on the issues. Through the inquiry learning, the scientific inquiry ability and innovative ability of students can be developed.

We have developed interactive learning tutorials for the theoretical physics courses. The interactive learning tutorial is designed to create an active learning environment in which students have a chance to correct their misconceptions, interpret the teaching contents, and build links between new knowledge and prior viewpoints. The tutorial development goes through a cyclical interactive process that includes research on student difficulties in learning theoretical physics concepts, followed by the development, evaluation and refinement of the instruction. During interactive learning, students engage in the topic via examples that focus their attention, explore the topic through facilitated questions and observation, explain what they have learned with the instructor facilitating further discussion to help refine their understanding, and extend what they have learned by applying the same concepts in different contexts (C. Singh, 2008). The guidance provided by the tutorials is decreased gradually.

\subsection{Establish virtual theoretical physics experiments}

The abstract and counterintuitive imagination of theoretical physics cannot be described in a coherent cognitive picture without the aid of information technology. Information technology can be used not only as an information management tool, but also as a means of teaching students of diverse information in theoretical physics teaching.

Computer simulation can be made with Maple, Matlab, Mathematica and other scientific computing software. Simulations can play an important role in creating virtual experiments and inquiry. Simulations give students the opportunity to experience a virtual physics world and interact with it. The virtual experiments can help students connect the mathematical abstraction to the real physical world they are trying to describe.

Problem based simulations allow students to monitor experiments, test new models and improve their intuitive understanding of complex physics phenomena (Sami, 2006). The basic strategy employed in theoretical physics class presented here is to create simple virtual experiments (such as compound pendulum, photoelectric effect, quantum bound states) that students program themselves, and then allow them to create a more sophisticated virtual of their own choosing (Todd D. Murphey, 2008). By allowing students to choose their own virtual experiments, their problem-solving capabilities and higher-order thinking abilities cane be significantly promoted.

\subsection{Improve assessment and appraisal methods, and promote students' all-round development}

Assessment and appraisal methods can provide sufficient information about how and why the student understands and misunderstands, and what directions the student must take to improve (Hattie, John, et al, 1998). Tests can perform feedback from students, but too often they are devoid of enough feedback to the students and thus can be an inefficient method for providing feedback. The simple evaluation approach has not been an effective method at informing teachers and students about what they have learned, and do not fully reflect the students' academic achievement.

In theoretical physics teaching, teachers can take advantage of comprehensive evaluation methods in order to enrich students' academic achievement and retain their previously learned subjects. There are several methods that may be employed by teachers in teaching theoretical physics. Those most frequently used are the tests, science and technological small thesis writing, courseware making, seminars, single subject research, and so on. When student's individualization is coupled or complemented with feedback, their achievement effects and scientific literacy would dramatically increase.

\section{Conclusion}

Theoretical physics instruction plays an important role in the research of basic science and training physics 
talents.

The priority of theoretical physics instruction is to stimulate the learning interest of students. In the teaching, teachers can stimulate students' learning interest by the creation of "cognitive conflict", and solve the conflict using suitable instruction methods. In the process of learning, students would grasp knowledge, develop thinking ability and perfect cognitive structure of theoretical.

The integration and optimization of theoretical physics courses can be progressed through the reduction of quantity and the choiceness of quality of the course contents.

Teachers can use integrated various teaching methods in order to achieve the best teaching effects. We have developed interactive learning tutorials for the theoretical physics courses. The interactive learning is designed to create an active learning environment for students.

Information technology can be used as a means of teaching students of diverse information in theoretical physics instruction. In theoretical physics instruction, computer simulation can be made with scientific computing software. Simulations give students the opportunity to observe virtual physics experiments. The virtual experiments can help students connect the mathematical abstraction to the real physical world they are trying to describe.

Theoretical physics teachers can take advantage of comprehensive evaluation methods, aim at enriching students' academic achievement. There are several methods that may be employed by teachers in theoretical physics teaching. Those most frequently used are the tests, science and technological small thesis writing, courseware making, seminars, single subject research, and so on.

\section{References}

C. Singh. (2008). Interactive Learning Tutorials on Quantum Mechanics. American Journal of Physics, 76(4\&5), 400-405.

Hattie, John, Jaeger \& Richard. (1998). Assessment and Classroom Learning: A Deductive Approach. Assessment in Education: Principles, Policy \& Practice, 5(1),111-122.

He, Ping \& Liao, An'sheng. (2000). Teaching Reform of Theoretical Physics. The Journal of Guangxi Normal University, 2,79-82.

He, Zuoxiu. (1993). Accelerating and Developing Research of Theoretical Physics. Science \& Technology Review, 5,20.

Lin, Cunzhen. (2000). Educational Objectives and Reform in the Teaching of Theoretical Physics. Physics, 29 (3), 183-184.

M. Born. (1941). The Teaching of Theoretical Physics in Universities. Reports on Progress in Physics ,8(1),1-10.

Mary, Stewart. (2002). Embedding Text-based Self-study into an HE Physics Course. Journal of Further and Higher Education, 26(3) ,251-262.

Muller R. \& Wiesner H. (2002). Teaching Quantum Mechanics on an Introductory Level. American Journal of Physics, 70(3),200-209.

Sami, Sahin. (2006). Computer Simulation in Science Education: Implications for Distance Education. Turkish Online Journal of Distance Education, 7 (4), 132-145.

Sun, Hai-bin \& Gong, Yan-xiang. (2007). Reform in the Teaching of Theoretical Mechanics. Higher Education of Sciences, 5, 93-96.

Todd D. Murphey. (2008). Teaching Rigid Body Mechanics Using Student-Created Virtual Environments. IEEE Transactions on Education,51(1), 45-52.

Wang, Jiao-guo. (2009). Physics Teaching Theory. XI'an: Shanxi Normal University Press, 79.

Wan, Yong. (2005). A Cognitive Analysis of the Major Problems with Teaching Reform of Theoretical Physics. Higher Education of Sciences, 6,86-89. 